\title{
Efecto de la secuencia anaeróbica-óxica-anóxica (AOA) en la eliminación de materia orgánica, fósforo y nitrógeno en un SBR modificado a escala de laboratorio
}

\author{
Effect of anaerobic-oxic-anoxic (AOA) sequence on the elimination of \\ organic matter, phosphorus and nitrogen in a modified SBR at laboratory scale
}

\author{
Mauricio Ramos Ramos ${ }^{1} \quad$ Juan F. Muñoz Paredes ${ }^{2 *} \quad$ Julio C. Saldarriaga Molina ${ }^{3}$ \\ Recibido 16 de julio de 2015, aceptado 8 de septiembre de 2016 \\ Received: July 16, 2015 Accepted: September 8, 2016
}

\begin{abstract}
RESUMEN
Se estudió el efecto de la secuencia anaeróbica, óxica, anóxica (AOA) en el funcionamiento de un reactor secuencial por tandas modificado a escala laboratorio, bajo condiciones de crecimiento biológico suspendido y adherido SAG-SBR (Suspended and Attached Growth Sequencing Batch Reactor), con el propósito de verificar la remoción simultánea de materia orgánica (MO) y de nutrientes (nitrógeno y fósforo). Se empleó un reactor de acrílico con un volumen útil de 10,2 litros, que integró un sistema de agitación, recirculación interna y aireación; se utilizó agua residual sintética (ARS), simulando un agua residual doméstica de concentración media y como medio de contacto se emplearon esferas y cilindros concéntricos de polipropileno. Durante la investigación, se empleó la secuencia AOA con diferentes ciclos y tiempos de duración ( $\mathrm{A}=15,5 \mathrm{~h} ; \mathrm{B}=13 \mathrm{~h} ; \mathrm{C}=11,5 \mathrm{~h}, \mathrm{D}=9,5 \mathrm{~h}$ ), aplicando al final la sedimentación del licor mezcla. Se realizó un estricto seguimiento de los parámetros de control más importantes del proceso como oxígeno disuelto (OD), $\mathrm{pH}$, temperatura y potencial de óxido reducción (ORP, por sus siglas en inglés) y se obtuvieron resultados significativos de remociones de los parámetros fisicoquímicos analizados, como demanda química de oxígeno (DQO), nitrógeno y fósforo, en particular con mayor eficiencia en las corridas efectuadas con los ciclos de menor tiempo de retención hidráulica. Es así, como se lograron eliminaciones en MO del orden del 97\%; en nitrógeno del $71 \%$ y en fósforo del $40 \%$. El ciclo con mejores resultados fue el ciclo D, con un tiempo total de 9,5 horas de duración y con una eliminación de MO del 96,6\%; en N del 70\% y en P del 51,2\%.
\end{abstract}

Palabras clave: Anaeróbica-óxica-anóxica (AOA), fósforo (P), materia orgánica (MO), nitrógeno (N), SBR.

\begin{abstract}
The effect of AOA (anaerobic, aerobic, anoxic) sequence was studied in the operation of a modified sequential batch reactor, at laboratory scale, under conditions of suspended and attached biological growth SAG-SBR, in order to verify the simultaneous removal of organic matter and nutrients (nitrogen and phosphorus). An acrylic reactor was used with a useful volume of 10,2 liters, that integrated a stirrer, internal recirculation and aeration system; synthetic sewage $(S W)$ was used for simulating a domestic

1 Universidad Mariana. Facultad de Ingeniería. Corporación Autónoma Regional de Nariño Corponariño. San Juan de Pasto, Nariño, Colombia. E-mail: mauramos@corponarino.gov.co; mauramos2000@yahoo.com

2 Universidad Mariana. Facultad de Posgrados y Relaciones Internacionales. San Juan de Pasto, Nariño, Colombia. E-mail: jfmunoz@umariana.edu.co; juanfemp@hotmail.com

3 Universidad de Antioquia. Facultad de ingeniería. Medellín, Antioquia, Colombia.

E-mail: julio.saldarriaga@udea.edu.co; julioc.saldarriaga@gmail.com

* Autor de correspondencia
\end{abstract}


wastewater of medium concentration and the means of contact were spheres and concentric cylinders of polypropylene. During the research, the AOA sequence was used with different cycles and duration times $(A=15.5 \mathrm{~h}, B=13 \mathrm{~h} ; C=11.5 \mathrm{~h}, \mathrm{D}=9.5 \mathrm{~h})$ applying at the end the sedimentation of mixture liquor. An accurate monitoring of the most important parameters of control process such as dissolved oxygen (DO), $\mathrm{pH}$, temperature and potential oxidation reduction was made and significant results were obtained in the analyzed physicochemical parameters as chemical demand oxygen (CDO), nitrogen and phosphorus, particularly with greater efficiency in lower hydraulic retention time. It is like that removals were achieved in MO the order of 97\%; $71 \%$ nitrogen and 40\% phosphorus. The cycle with the best performing was D, with a total time of 9.5 hours and a removal of $96.6 \%$ of $M O ; 70 \% N$ and $51.2 \% P$.

Keywords: Anaerobic-oxic-anoxic (AOA), phosphorus (P), organic matter (MO), nitrogen (N), SBR.

\section{INTRODUCCIÓN}

En el tratamiento de las aguas residuales (AR), si bien es cierto se han desarrollado innumerables tecnologías para la remoción de contaminantes, en la práctica, en países en vías de desarrollo quizá el principal objetivo de las plantas de tratamiento de aguas residuales es la remoción de materia orgánica (MO) y sólidos, sin mayor énfasis en la eliminación de nutrientes, que en gran medida son sustancias causantes del proceso de eutrofización de los cuerpos de agua y limitan sus usos por la presencia de compuestos hasta niveles de alerta en toxicidad [1].

La presencia de compuestos de nitrógeno $(\mathrm{N})$ y fósforo (P) es esencial para el crecimiento de las plantas y de otros organismos, por lo que se les llama de forma genérica nutrientes. El vertido de aguas residuales ricas en estos compuestos puede dar lugar al proceso conocido como eutrofización, el que se entiende como un proceso complejo de fertilización de las aguas naturales con sustancias nutritivas, especialmente $\mathrm{N}$ y $\mathrm{P}$ en formas asimilables por la vegetación, que origina un acelerado crecimiento de algas y plantas acuáticas [2], un incremento de la productividad en todos los niveles de la cadena alimentaria y un empeoramiento progresivo de las características fisicoquímicas del agua [3]. Asimismo, es un proceso que puede ser irreversible y que se presenta en los ecosistemas acuáticos lénticos como respuesta al incremento abundante en la densidad de las comunidades de fitoplancton [4].

$\mathrm{Al}$ igual que la $\mathrm{MO}$, la presencia de $\mathrm{P}$ y $\mathrm{N}$ en las descargas de aguas residuales genera una disminución drástica de los niveles de oxígeno disuelto (OD) en los cuerpos de agua receptores, debido a la oxidación bacteriana del amonio a nitrato [5], fenómeno que origina condiciones adversas para el sostenimiento de los ecosistemas, particularmente la generación de un ambiente tóxico para los organismos acuáticos; tal es el caso particular del nitrógeno inorgánico, representado como la suma de amonio ionizado $\left(\mathrm{NH}_{4}{ }^{+}\right)$y no ionizado $\left(\mathrm{NH}_{3}\right)$, el nitrito $\left(\mathrm{NO}_{2}^{-}\right)$y el nitrato $\left(\mathrm{NO}_{3}{ }^{-}\right)$, el que tiene reconocidos efectos tóxicos sobre las especies hidrobiológicas [6].

Adicionalmente, para el ser humano la ingesta de nitritos y nitratos, puede inducir efectos adversos en la salud, generando metahemoglobinemia en bebés [7], así como también, la ingesta prolongada de estos iones puede contribuir al desarrollo de linfomas y cánceres, enfermedades coronarias, infecciones del tracto respiratorio, y malformaciones en los recién nacidos [8]. Por otra parte, el exceso de compuestos asimilables de fósforo puede ocasionar en la salud humana hipocalcemia y problemas de riñones [9]. Este fenómeno ha sido identificado como la principal causa de deterioro de la calidad del agua, que puede restringir su uso para pesca, recreación, industrial y de consumo, tal como lo advierte la Agencia de Protección Ambiental de los Estados Unidos [10].

Las aguas residuales domésticas al igual que muchos residuos líquidos industriales, presentan importantes contenidos de $\mathrm{N}$ y $\mathrm{P}$, en particular los sectores de la industria alimenticia, las aguas residuales urbanas y las provenientes del sector pecuario, entre otras [11]. Los procesos de eliminación de nutrientes son más complejos que los de remoción de materia orgánica (Demanda Bioquímica de Oxígeno, DBO); para su eliminación se requiere la combinación de por lo menos dos etapas: aeróbica y anóxica en el caso de N [12-13] y anaeróbica y aeróbica en el caso del P [14]. 
Esta investigación fue motivada por la necesidad de evaluar nuevas condiciones de operación de esta tecnología, para la eliminación simultánea de $\mathrm{MO}, \mathrm{N}$ y $\mathrm{P}$, contaminantes que generan deterioro del recurso hídrico y, por consiguiente, riesgo a la salud pública. Para tal efecto, bajo este contexto fue considerada la tecnología SBR para ser probada bajo un diseño particular soportado en la combinación de tratamiento biológico suspendido y adherido en la secuencia anaeróbica-óxica-anóxica (AOA), proceso innovador que a su vez presenta aportes en el campo del tratamiento de aguas residuales con tecnologías de bajo costo junto a otras investigaciones similares llevadas a cabo por diferentes autores [5, 15-17].

\section{METODOLOGÍA}

\section{Instrumentación y equipos}

Se empleó un reactor cilíndrico de acrílico con un volumen útil de 10,2 litros, con las correspondientes válvulas y conexiones para la alimentación y descarga del agua residual. El sistema de agitación consistió en dos dispositivos rotatorios con cuatro paletas cada uno, dispuestas a $45^{\circ}$ con respecto a su eje y con un área total de 149,5 cm²; el sistema de aireación consistió en placas porosas de burbuja fina con gránulos de $\mathrm{SiO}_{2}$ ubicadas en el fondo del reactor y un sistema de recirculación interna de licor mezcla a una tasa de $1,2 \mathrm{l} / \mathrm{min}$.

En la Figura 1, se puede apreciar el reactor SBR, dentro del que, como material de contacto se emplearon esferas (móviles y fijas) y cilindros concéntricos de polipropileno con las características que se presentan en la Tabla 1. Las esferas o biobolas fijas (24 unidades) fueron atadas por pares en vertical $(6 \mathrm{~cm})$ sobre un aro de polietileno semirrígido, que se ancló a una

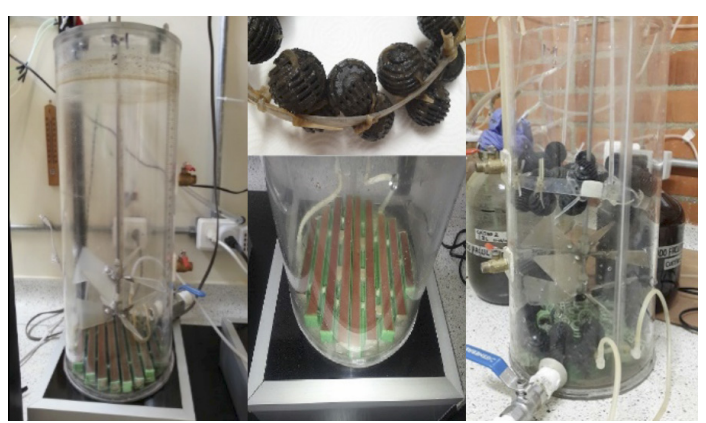

Figura 1. Detalles constructivos del SBR a escala laboratorio. altura de $25 \mathrm{~cm}$ desde la superficie del sistema de aireación. Es preciso anotar que el aro junto con las esferas fue adaptado para desplazarse hasta el fondo durante la fase de sedimentación, de tal forma que no interviniese con la calidad del agua residual clarificada. Las esferas restantes y los cilindros fueron dispuestos libremente para estar en contacto por todo el volumen del reactor con el licor mezcla.

Tabla 1. Características de los medios de contacto.

\begin{tabular}{|c|c|}
\hline \multicolumn{2}{|l|}{ Biobolas } \\
\hline Parámetro & Valor \\
\hline Diámetro $(\mathrm{cm})$ & 3 \\
\hline Volumen $\left(\mathrm{cm}^{3}\right)$ & 14,14 \\
\hline Masa (g) & 8,96 \\
\hline Densidad $\left(\mathrm{g} / \mathrm{cm}^{3}\right)$ & 0,97 \\
\hline $\begin{array}{l}\text { Número total de unidades libres y fijas } \\
\text { (24 fijas, } 13 \text { libres) }\end{array}$ & 37 \\
\hline $\begin{array}{l}\text { Área superficial específica de contacto por } \\
\text { unidad }\left(\mathrm{cm}^{2} / \mathrm{cm}^{3}\right)\end{array}$ & 7,67 \\
\hline \multicolumn{2}{|l|}{ Cilindros } \\
\hline Diámetro interno $(\mathrm{cm})$ & 2 \\
\hline Diámetro externo $(\mathrm{cm})$ & 2,2 \\
\hline Altura $(\mathrm{cm})$ & 1,3 \\
\hline Volumen cilindro $\mathrm{cm}^{3}$ & 3,47 \\
\hline Masa $(\mathrm{g})$ & 4 \\
\hline Densidad $\left(\mathrm{g} / \mathrm{cm}^{3}\right)$ & 1,15 \\
\hline Número de unidades libres & 33 \\
\hline $\begin{array}{l}\text { Área superficial específica de contacto por } \\
\text { unidad }\left(\mathrm{cm}^{2} / \mathrm{cm}^{3}\right)\end{array}$ & 4,53 \\
\hline
\end{tabular}

En la Figura 2 se observa la forma de los medios de contacto empleados en el SBR en condiciones fijas y móviles.

El montaje del sistema se ubicó en las instalaciones de la sede Alvernia de la Universidad Mariana

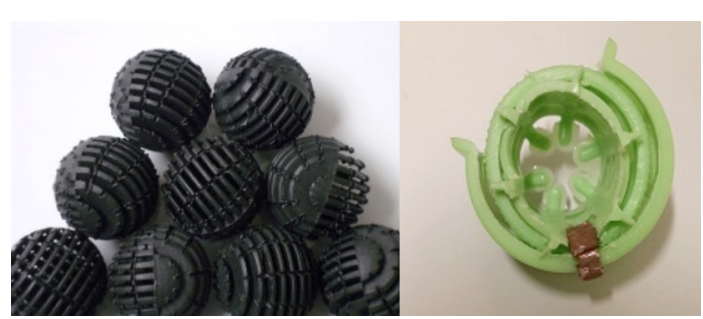

Figura 2. Medios de contacto empleados en el reactor SAG-SBR, biobolas y cilindros. 
de San Juan de Pasto, adaptando en su medida la automatización posible a esta escala. El seguimiento instrumental y el desarrollo continuo de los muestreos y análisis se realizaron con base en las técnicas del Método Estándar de Análisis de Aguas y Aguas Residuales, versión 21 [18].

Los parámetros fisicoquímicos monitoreados tanto para el ARS como para las muestras tomadas en los diferentes ciclos del tratamiento fueron los siguientes: Demanda Química de Oxígeno (DQO), Nitritos $\left(\mathrm{NO}_{2}^{-}\right)$, Nitratos $\left(\mathrm{NO}_{3}{ }^{-}\right)$, NTK, fósforo total (PT), sólidos totales (ST), sólidos suspendidos totales (SST) y sólidos suspendidos volátiles (SSV).

Para tener un adecuado seguimiento del proceso, se evaluaron continuamente los parámetros de control más importantes, como $\mathrm{pH}$, temperatura, OD y ORP. Los electrodos de las sondas correspondientes se ubicaron en la parte superior del reactor, mediante tres orificios independientes, siempre sumergidos en el licor mezcla durante cada ciclo de tratamiento. Estos fueron sometidos a limpieza al finalizar cada corrida, con el propósito de garantizar la lectura real y representativa de los parámetros de control del proceso.

\section{Preparación del agua residual sintética (ARS)}

El ARS, se preparó simulando un agua residual doméstica de concentración media, de acuerdo con lo recomendado en [19]. Se prepararon semanalmente dos lotes de ARS con un volumen de 60 litros cada uno, con el fin de contar con la cantidad suficiente para la realización de las corridas en cada ciclo $\mathrm{y}$ para el funcionamiento permanente del reactor durante siete días. El sustrato fue alimentado al reactor por una bomba peristáltica a una tasa de 0,6 1/min, (50 rpm). En la Tabla 2 se identifica la composición del agua residual sintética, indicando las cantidades para un volumen de agua de 60 litros.

Tabla 2. Composición del agua residual sintética (ARS).

\begin{tabular}{|l|c|c|}
\hline Compuesto & Unidad & Cantidad para 60 litros \\
\hline $\mathrm{C}_{2} \mathrm{H}_{3} \mathrm{NaO}_{2}$ & g & 31,80 \\
\hline $\mathrm{CH}_{3} \mathrm{COOH}$ & ml & 14,40 \\
\hline $\mathrm{CH}_{4} \mathrm{~N}_{2} \mathrm{O}$ & g & 5,60 \\
\hline $\mathrm{K}_{2} \mathrm{HPO}_{4}$ & g & 2,50 \\
\hline $\mathrm{NaCl}$ & g & 0,42 \\
\hline $\mathrm{CaCl}_{2} \cdot 2 \mathrm{H}_{2} \mathrm{O}$ & $\mathrm{g}$ & 0,24 \\
\hline $\mathrm{Mg}_{2} \mathrm{SO}_{4} \cdot 7 \mathrm{H}_{2} \mathrm{O}$ & $\mathrm{g}$ & 0,12 \\
\hline
\end{tabular}

Para suplir los requerimientos micronutricionales, se empleó una solución de sustancias traza para la preparación del ARS, siguiendo la composición que se presenta en la Tabla 3. Una vez preparada esta solución, se añadieron $3 \mathrm{ml}$ por cada litro de ARS preparada [20].

Tabla 3. Composición de la solución de sustancias traza.

\begin{tabular}{|l|c|}
\hline \multicolumn{1}{|c|}{ Compuesto } & Cantidad (g) para 1 litro \\
\hline $\mathrm{FeCl}_{3} \cdot 6 \mathrm{H}_{2} \mathrm{O}$ & 1,50 \\
\hline $\mathrm{H}_{3} \mathrm{BO}_{3}$ & 0,15 \\
\hline $\mathrm{CuSO}_{4} \cdot 5 \mathrm{H}_{2} \mathrm{O}$ & 0,03 \\
\hline $\mathrm{KI}$ & 0,18 \\
\hline $\mathrm{MnCl}_{2} \cdot 4 \mathrm{H}_{2} \mathrm{O}$ & 0,12 \\
\hline $\mathrm{Na}_{2} \mathrm{Mo}_{4} \cdot 2 \mathrm{H}_{2} \mathrm{O}$ & 0,06 \\
\hline $\mathrm{ZnSO}_{4} \cdot 7 \mathrm{H}_{2} \mathrm{O}$ & 0,12 \\
\hline $\mathrm{CoCl}_{2} \cdot 6 \mathrm{H}_{2} \mathrm{O}$ & 0,15 \\
\hline
\end{tabular}

\section{Inoculación y siembra del lodo biológico}

El inóculo (siembra de microorganismos) fue obtenido de una planta de tratamiento de aguas residuales domésticas, de aireación extendida del municipio de Chachagüi, Departamento de Nariño, Colombia. Se recolectó en iguales proporciones y en combinación, lodo activado del tanque de sedimentación y del tanque de aireación, con el propósito de mejorar las características físicas y metabólicas de la biomasa activa. Posteriormente, esta suspensión se mezcló con lodo proveniente de una laguna facultativa del sistema de tratamiento de aguas residuales de una central de sacrificio de ganado, en una proporción 70/30, respectivamente.

Cabe destacar que el influente a esta laguna, combina las aguas residuales domésticas provenientes de esta misma central, por lo que sus lodos ya se encuentran adaptados al tratamiento de sustrato combinado residual doméstico y de origen pecuario con alto contenido de nitrógeno, condición que le aportó al inóculo biomasa facultativa de metabolismo fermentativo y respiratorio, vital para la descomposición de la materia orgánica y eliminación de $\mathrm{N}$ y P de manera simultánea, frente a los cambios cíclicos de tratamiento con y sin la presencia de oxígeno, presentados en el desarrollo de la secuencia anaeróbica, óxica y anóxica del SBR posteriormente evaluado.

El proceso de cultivo en recipientes de vidrio de 3 litros, requirió 6 meses, previo a la carga, arranque 
y estabilización del reactor SAG-SBR, tiempo durante el que se elaboraron ensayos semanales, es así como para los dos últimos meses se logró obtener una mezcla homogénea y de concentración adecuada, con un promedio de $3.287 \mathrm{mg} / \mathrm{l}$ de sólidos suspendidos volátiles, remociones superiores al 93\% de DQO y un IVL ubicado en un intervalo entre 94 y $97 \mathrm{~g} / \mathrm{ml}$, parámetros que demostraron el buen comportamiento del proceso de aclimatación del biolodo en los recipientes de vidrio, en condiciones de laboratorio. Los medios de contacto fueron integrados al mes de iniciados los cultivos en un total de 37 biobolas y 33 cilindros. Se precisa que esta mezcla se sometió a secuencias programadas de tiempos de aireación (ON) de 210 min y no aireación (OFF) de $270 \mathrm{~min}$, incluyendo una hora de sedimentación en condiciones de reposo, por medio de un temporizador. Se alimentaron inicialmente 2 litros de lodo con $250 \mathrm{ml} / \mathrm{d}$ de ARS por dos semanas. Posteriormente se incrementó la cantidad de ARS a $400 \mathrm{ml} / \mathrm{d}$ por dos semanas más. Finalmente, se estableció la alimentación de ARS en $1000 \mathrm{ml} / \mathrm{d}$ desde la quinta semana en adelante manteniendo una relación F/M de $0,043 \mathrm{~d}^{-1}$. Desde la tapa hermética de estos recipientes se instaló un mecanismo de agitación, mezcla y aireación con microburbujas, asegurando la alimentación diaria de 1 L de ARS y la mezcla de la suspensión, el retiro de lodos de exceso $(\sim \theta \mathrm{c}=10 \mathrm{~d})$ y el retiro del clarificado, de tal forma que la biomasa durante los 6 meses previos, se adaptó gradualmente al sustrato sintético (ARS), a la secuencia anaeróbica -óxicaanóxica-sedimentación y al régimen de trabajo que posteriormente requirió el SAG-SBR, durante el arranque y estabilización, proceso que se describe a continuación.

\section{Arranque y estabilización del SAG-SBR}

Con el lodo madurado al sexto mes, y considerando la adaptación de las biomasas en los recipientes de vidrio anteriormente descritos, se procedió a dar inicio en un período aproximado de 2,5 meses, al proceso de arranque y estabilización del reactor SAG-SBR; para esto se tuvieron en cuenta las condiciones más importantes del proceso, las cuales se presentan en la Tabla 4.

Inicialmente se arrancó el reactor SG-SBR, vertiendo el contenido de 3,37 L de lodo adaptado y se completó con $6,84 \mathrm{~L}$ de ARS por cada ciclo, para un total de 10,21 litros, volumen útil del SAG-SBR. De esta forma
Tabla 4. Condiciones de arranque del SAG-SBR.

\begin{tabular}{|c|c|}
\hline Etapa & Detalle \\
\hline Carga de sustrato & $\begin{array}{l}\text { Q de Sustrato=0,6 1/min. Aireación } \\
\text { Off. Mezcla ON a } 4 \mathrm{rpm} \text {. Recirculación } \\
\text { interna ON a 1,2 1/min. Duración etapa: } \\
14 \mathrm{~min} . \mathrm{F} / \mathrm{M} \text { de } 0,18 \mathrm{~d}^{-1} \text {. }\end{array}$ \\
\hline Ciclo Anaerobio & $\begin{array}{l}\text { Aireación Off - OD en 0,01-0,02 mg/l. } \\
\text { Mezcla ON a } 4 \text { rpm. Recirculación } \\
\text { ON a 1,2 1/min. } \\
\text { Duración de la etapa: } 2,5 \mathrm{~h}\end{array}$ \\
\hline Ciclo Aerobio & $\begin{array}{l}\text { Aireación ON - OD entre } 2 \text { y } 3 \mathrm{mg} / \mathrm{l} \text {. } \\
\text { Mezcla ON a } 4 \mathrm{rpm} \text {. Recirculación } \\
\text { ON a } 1,2 \text { 1/min } \\
\text { Duración de la etapa: } 3,5 \mathrm{~h}\end{array}$ \\
\hline Ciclo Anóxico & $\begin{array}{l}\text { Aireación Off- OD en 0,01- 0,02 mg/l. } \\
\text { Mezcla ON a } 4 \text { rpm. Recirculación } \\
\text { ON a 1,2 1/min } \\
\text { Duración de la etapa: } 4 \text { h }\end{array}$ \\
\hline Purga de Lodo & $\begin{array}{l}\text { Aireación Off. Mezcla ON a } 4 \text { rpm. } \\
\text { Eliminación de aproximadamente } \\
1 \text { Litro de LM. Tiempo medio de } \\
\text { retención celular } \theta \text { c en } 10 \text { días. } \\
\text { Actividad inmediata. }\end{array}$ \\
\hline Sedimentación & $\begin{array}{l}\text { Aireación OFF. Mezcla Off. } \\
\text { Recirculación OFF. Duración de la } \\
\text { etapa: } 1 \mathrm{~h}\end{array}$ \\
\hline Descarga & $\begin{array}{l}\text { Descarga del clarificado a Caudal } \\
\text { de } 0,5 \mathrm{l} / \mathrm{min} \text {, evitando resuspensión } \\
\text { de sólidos del fondo. Duración de la } \\
\text { etapa: } 10 \mathrm{~min} \text {. }\end{array}$ \\
\hline
\end{tabular}

se logró adaptar el proceso biológico en secuencia AOA al diseño propio del reactor SAG-SBR, previo a la realización de las corridas finales de evaluación, tal como se describe en la sección de Resultados.

\section{Ciclos de operación y tiempos de retención hidraúlico del SAG-SBR}

Se utilizaron cuatro (4) ciclos de trabajo en el reactor, estableciendo diferentes tiempos de retención hidráulico (TRH) de las fases anaeróbicaaeróbica-anóxica-sedimentación. Se llevó a cabo la evaluación de un ciclo por semana, tiempo en el que se analizaron las muestras de dos corridas por ciclo, con las que se registraron parámetros de control como T, pH, OD y ORP y parámetros fisicoquímicos para medir la eficiencia de las fases AOA y del sistema SAG-SBR en general, en términos de eliminación de MO, $\mathrm{N}$ y $\mathrm{P}$.

En la Tabla 5 se presentan los ciclos utilizados conformados por diferentes TRH, incluyendo la etapa final de sedimentación. 
Tabla 5. Ciclos de Operación del SAG-SBR.

\begin{tabular}{|c|c|c|c|c|c|}
\hline \multirow[b]{2}{*}{ 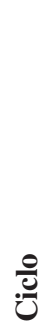 } & \multicolumn{4}{|c|}{ Duración de cada fase } & \multirow[b]{2}{*}{ 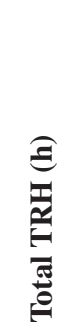 } \\
\hline & 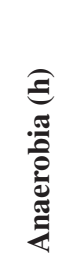 & 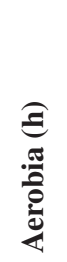 & 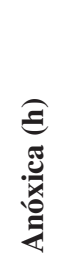 & 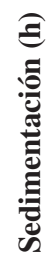 & \\
\hline $\mathrm{A}$ & 3 & 5,5 & 6 & 1 & 15,5 \\
\hline $\mathrm{B}$ & 3 & 4 & 5 & 1 & 13 \\
\hline $\mathrm{C}$ & 2,5 & 3,5 & 4,5 & 1 & 11,5 \\
\hline $\mathrm{D}$ & 2 & 3 & 3,5 & 1 & 9,5 \\
\hline
\end{tabular}

\section{RESULTADOS}

\section{Comportamiento del reactor SAG-SBR en fase} de arranque y estabilización

En un período aproximado de 2,5 meses, se llevó a cabo el proceso de arranque y estabilización, inicialmente para un ciclo intermedio de $11 \mathrm{~h}$ y posteriormente para uno de $13 \mathrm{~h}$; para ese último, se registraron los parámetros de control y se realizaron pruebas para evaluar la evolución en la eliminación simultánea de MO, N y P. En la Figura 3, se registra el comportamiento del ORP y $\mathrm{pH}$ durante la estabilización; en esta figura se ubica el punto de inflexión en el tiempo aproximado de 260 minutos en fase aerobia, a valores cercanos a $50 \mathrm{mV}$ y en un $\mathrm{pH}$ de 7,75 unidades, punto que indica el final de la nitrificación donde la concentración de NTK fue cercana a cero $(0,0)$.

También se destaca la buena propiedad de sedimentabilidad de los lodos, reflejada en un IVL entre 91,3 y 93,7 y el intervalo de trabajo de la temperatura en las fases AOA, tal como se indica en la Figura 4, donde se registraron IVL inferiores a $94 \mathrm{ml} / \mathrm{g}$ (lodo excelente) y valores de T y OD dentro del intervalo recomendado en investigaciones con SBR de $19-27^{\circ} \mathrm{C}$ y de $1,5-4 \mathrm{mg} / \mathrm{l}$, respectivamente [21-22].

Durante la etapa de estabilización fue evidente la interacción del crecimiento biológico suspendido y adherido, quienes se desarrollaron en el mismo reactor en todas las fases AOA; se generó biopelícula en los medios de contacto empleados, con la suficiente adherencia y consistencia para soportar los flujos

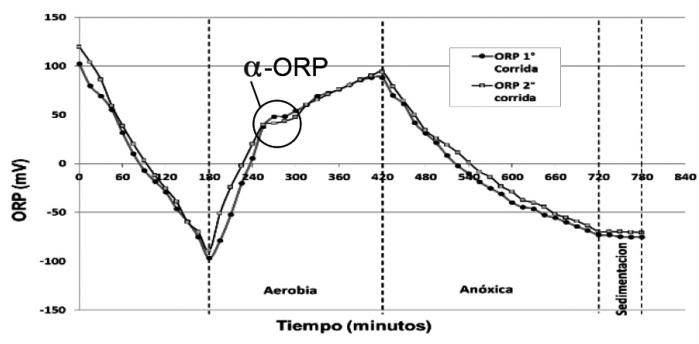

(a)

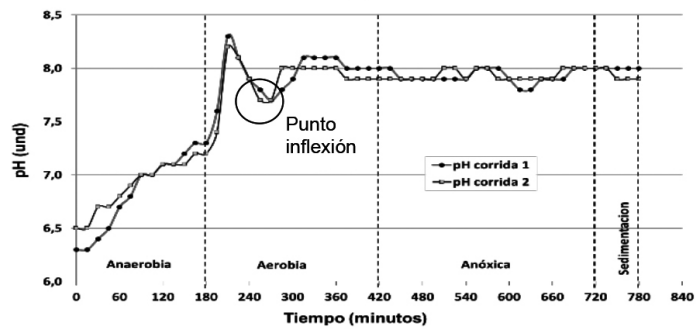

(b)

Figura 3. Variación de parámetros de control. (a) ORP; (b) pH, estabilización SAG-SBR.

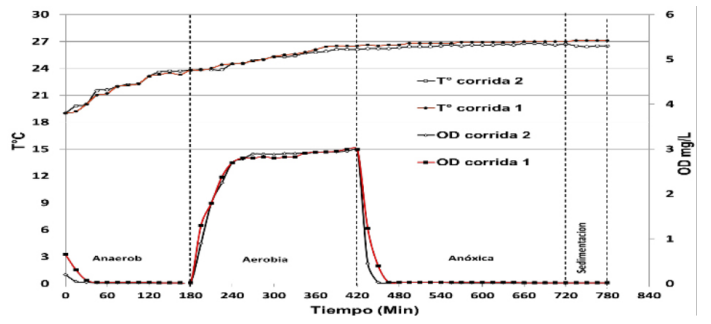

(a)

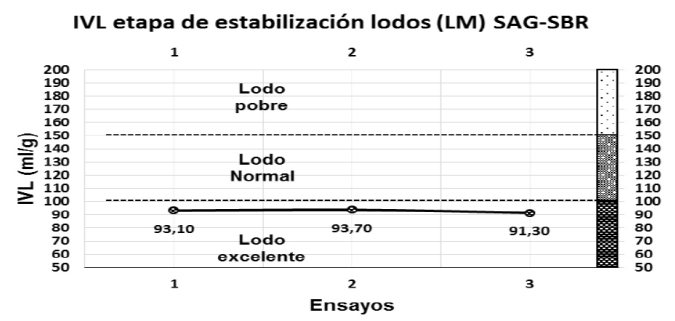

(b)

Figura 4. (a) Variación de T y OD; (b) IVL etapa de estabilización SAG-SBR.

internos durante el funcionamiento del sistema de mezclado, aireación y recirculación del licor mezcla, en particular para aquellos que se desplazaron libremente por todo el volumen del reactor.

En la Figura 5, se observa el comportamiento de la DQO, P, NTK, $\mathrm{NO}_{3}{ }^{-}$y $\mathrm{NO}_{2}{ }^{-}$en el SAG-SBR. La MO 
ingresó con $526 \mathrm{mg}$ DQO/l para dos corridas de $13 \mathrm{~h}$ de duración y se registraron al final concentraciones de 16 y $13 \mathrm{mg}$ DQO/l, representando eliminaciones de MO de $96,94 \%$ y $97,53 \%$ respectivamente. Asímismo, la concentración de NTK descendió en fases anaerobia y aerobia, hasta niveles cercanos a cero (0), que nuevamente se constituye en un indicador de la presencia de bacterias nitrificantes con un buen desempeño metabólico; situación que a su vez se ve soportada por la alta concentración de $\mathrm{NO}_{3}{ }^{-}$superior a 100 y $120 \mathrm{mg} / \mathrm{l}$ para las dos corridas de prueba y bajas concentraciones de $\mathrm{NO}_{2}{ }^{-}$inferiores a $0,021 \mathrm{mg} / \mathrm{l}$.

En lo que concierne al comportamiento del $\mathrm{P}$, en la Figura 5b se observa, que una vez liberados los ortofosfatos en la fase anaerobia, se obtuvo extra asimilación de estos por los organismos acumuladores de $\mathrm{P}$ (PAO, por sus siglas en inglés) en forma de polifosfatos intracelulares, con la reducción de su concentración hasta niveles de $6 \mathrm{mg} / \mathrm{l}$ y evidenciándose remociones con tendencia a incrementarse, como las registradas en el segundo ensayo con un porcentaje de eliminación de 34,13\%.

Complementariamente en la Figura 6, y con base en el balance de masa correspondiente, se observa buen comportamiento en la remoción de NT durante la estabilización del SAG-SBR, condición que propició el desarrollo de una biopelícula, capaz de albergar preferentemente bacterias nitrificantes y desnitrificantes para la eliminación del N presente en la ARS [23]; tal es así que durante esta fase, se observaron niveles de remoción que progresivamente pasaron de $60,81 \%$ a $63,25 \%$, registrados en dos ensayos realizados para la misma secuencia AOA.

Asímismo, con los sólidos representados en los SSV, conforme a su alta sedimentabilidad (véase Figura 4b y 6b), se logró una remoción superior al $99 \%$, en los ensayos realizados.

\section{Comportamiento del reactor SAG-SBR en fase de operación normal}

El comportamiento de los parámetros de control registrados in situ en etapa de operación normal, siguió una tendencia similar a la fase de arranque y estabilización, como se puede apreciar en la Figura 7. En esta figura se presentan los valores monitoreados durante la corrida del ciclo $\mathrm{D}$, la cual

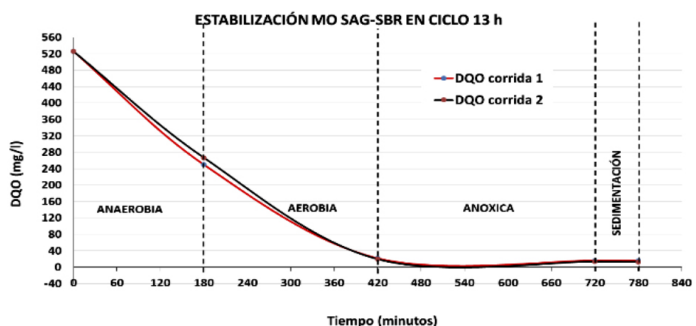

(a)

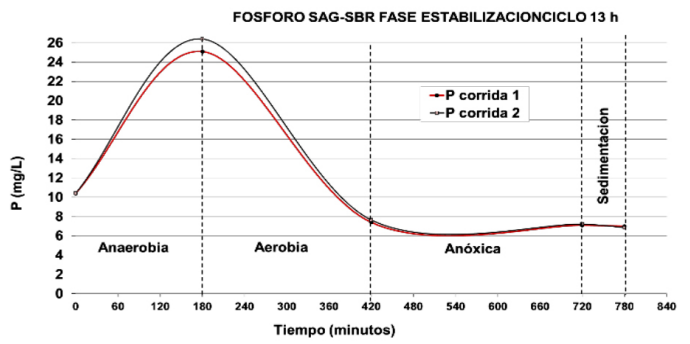

(b)

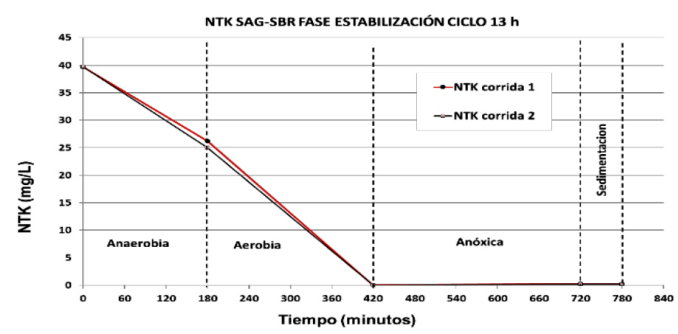

(c)

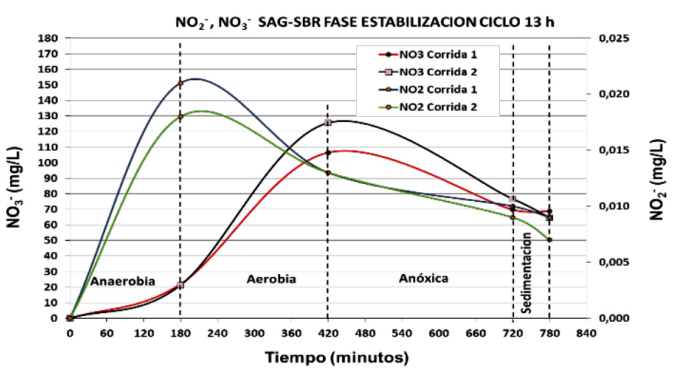

(d)

Figura 5. Variación de los parámetros. (a) DQO, (b) $\mathrm{P}$, (c) $\mathrm{NTK}$, (d) $\mathrm{NO}_{3}^{-}{\mathrm{y} \mathrm{NO}_{2}}^{-}$ estabilización de SAG-SBR

suministró los mejores resultados en términos de eliminación simultánea de MO, P y N.

Los parámetros ORP y $\mathrm{pH}$ se comportaron de manera similar en todos los ciclos de la fase de operación normal. El ORP descendió en las fases anaeróbica y anóxica, y ascendió en la fase aeróbica 


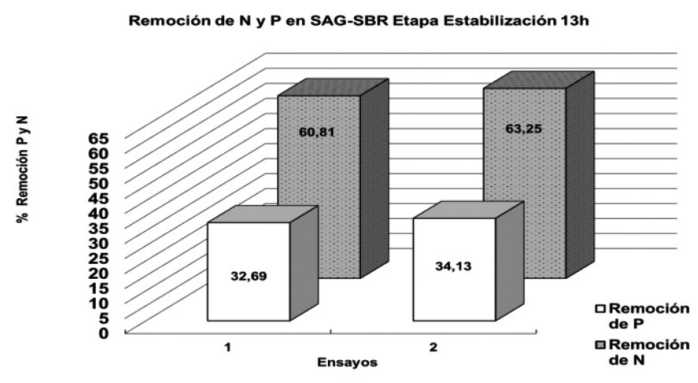

(a)

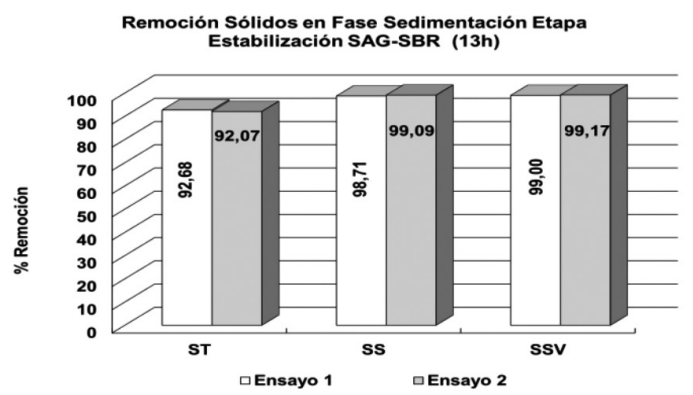

(b)

Figura 6. (a) Remoción de N y P, (b) Remoción de sólidos estabilización de SAG-SBR.

hasta presentar un punto de inflexión denominado $\alpha$ ORP, el que coincide con el punto de inflexión de la curva de $\mathrm{pH}$, indicadores de que la nitrificación ha finalizado [14]. De acuerdo con los resultados de este ciclo, se utilizaron tiempos cortos para la nitrificación, aproximadamente de 195 minutos, cuando se observó el punto de quiebre alcanzando los $18 \mathrm{mV}$ y 7,75 unidades de $\mathrm{pH}$, respectivamente.

La temperatura y el OD tuvieron un comportamiento similar en todos los ensayos de la fase de operación normal, en la Figura 7c se puede observar que la temperatura se encuentra en el rango entre 19 y $28^{\circ} \mathrm{C}$, y el OD entre 0,02 y $3 \mathrm{mg} / \mathrm{l}$, valores recomendados para el funcionamiento de este tipo de reactores [15-16, 21].

En todos los ciclos evaluados, se evidenció la disminución de MO, N y P en etapa de operación normal, con respecto a la muestra del sustrato inicial; no obstante, fue superior la eficiencia de eliminación de estos contaminantes en el ciclo D del reactor SAG-SBR, en el que se utilizaron los tiempos de duración más reducidos en las fases AOA con un total de 9,5 horas, representadas en $2 \mathrm{~h}$ de fase anaeróbica, $3 \mathrm{~h}$ en fase óxica, 3,5 h en fase anóxica y una hora de sedimentación.

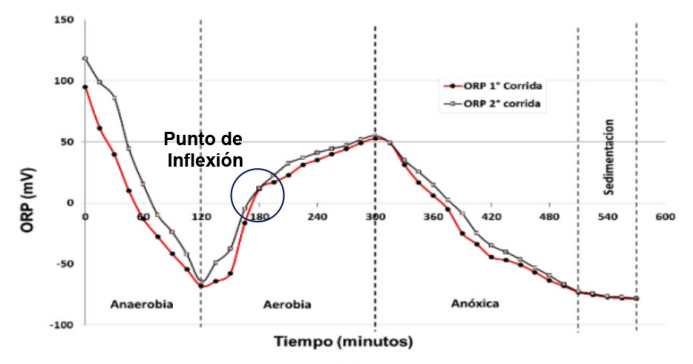

(a)

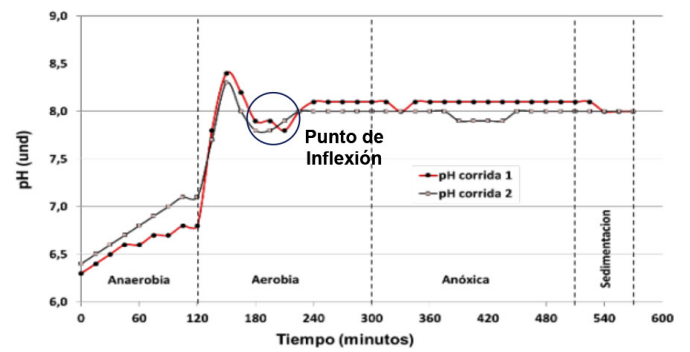

(b)

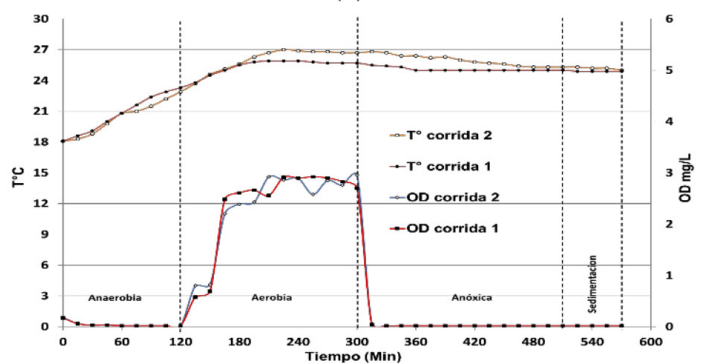

(c)

Figura 7. Variación de parámetros de control. (a) ORP; (b) pH; (c) T y OD en ciclo "D" del SAG-SBR.

En la Tabla 6 se observan los porcentajes de eliminación obtenidos para MO, P, NTK y el comportamiento $\mathrm{NO}_{2}{ }^{-}$y $\mathrm{NO}_{3}{ }^{-}$para cada ciclo de tratamiento.

Con base en los resultados obtenidos, en términos de concentración de MO, se observó que la DQO, se elimina independientemente del ciclo empleado; con un valor inicial promedio de $520 \mathrm{mg} / \mathrm{l}$ se reduce sustancialmente en las dos primeras fases anaeróbica y aeróbica hasta valores de $9,6 \mathrm{mg} / \mathrm{l}$ y $16,0 \mathrm{mg} / \mathrm{l}$ para el ciclo B y C, respectivamente. El porcentaje de eliminación es superior al $96 \%$, valor concordante con estudios similares en este tipo de reactores [5, 15-16].

Las concentraciones de MO al finalizar la fase aerobia son determinantes para garantizar disponibilidad de 
Tabla 6. Eliminación de MO, P y NTK y comportamiento de $\mathrm{NO}_{2}^{-}$y $\mathrm{NO}_{3}{ }^{-}$por ciclo SAG-SBR en operación normal.

\begin{tabular}{|c|c|c|c|c|}
\hline \multirow{3}{*}{ Fase } & \multicolumn{4}{|c|}{ MO SAG-SBR (mg/l) } \\
\hline & \multicolumn{4}{|c|}{ Ciclo } \\
\hline & A & B & C & D \\
\hline Sustrato ARS & 520,00 & 520,00 & 520,00 & 520,00 \\
\hline Anaerobia & 267,20 & 256,60 & 240,00 & 268,80 \\
\hline Aerobia & 11,20 & 9,60 & 16,00 & 12,80 \\
\hline Anóxica & 27,20 & 9,60 & 16,00 & 17,60 \\
\hline Sedimentación & 19,20 & 9,60 & 16,00 & 17,60 \\
\hline Eliminación $(\%)$ & 96,3 & 98,1 & 96,9 & 96,6 \\
\hline \multicolumn{5}{|c|}{ P SAG-SBR (mg/l) } \\
\hline Sustrato ARS & 9,95 & 10,05 & 10,01 & 10,04 \\
\hline Anaerobia & 14,75 & 23,90 & 20,51 & 20,74 \\
\hline Aerobia & 6,49 & 7,57 & 5,44 & 4,91 \\
\hline Anóxica & 7,80 & 6,32 & 5,82 & 4,74 \\
\hline Sedimentación & 6,54 & 6,69 & 5,99 & 4,89 \\
\hline Eliminación (\%) & 34,22 & 33,38 & 40,14 & 51,27 \\
\hline \multicolumn{5}{|c|}{ NTK SAG-SBR (mg/l) } \\
\hline Sustrato ARS & 39,57 & 39,77 & 39,96 & 40,04 \\
\hline Anaerobia & 30,95 & 18,51 & 26,64 & 30,34 \\
\hline Aerobia & 0,59 & 0,00 & 0,51 & 0,61 \\
\hline Anóxica & 1,88 & 0,91 & 1,02 & 0,61 \\
\hline Sedimentación & 1,88 & 1,60 & 0,51 & 0,61 \\
\hline Eliminación (\%) & 95,25 & 95,98 & 98,72 & 98,48 \\
\hline \multicolumn{5}{|c|}{$\mathrm{NO}_{2}^{-} \mathrm{SAG}-\mathrm{SBR}(\mathrm{mg} / \mathrm{l})$} \\
\hline Sustrato ARS & 0,000 & 0,000 & 0,000 & 0,000 \\
\hline Anaerobia & 0,014 & 0,017 & 0,030 & 0,008 \\
\hline Aerobia & 0,076 & 0,014 & 0,134 & 0,310 \\
\hline Anóxica & 0,073 & 0,014 & 0,006 & 0,008 \\
\hline Sedimentación & 0,074 & 0,013 & 0,006 & 0,005 \\
\hline \multicolumn{5}{|c|}{$\mathrm{NO}_{3}{ }^{-} \mathrm{SAG}-\mathrm{SBR}(\mathrm{mg} / \mathrm{l})$} \\
\hline Sustrato ARS & 0,00 & 0,00 & 0,00 & 0,00 \\
\hline Anaerobia & 16,60 & 16,18 & 8,35 & 11,59 \\
\hline Aerobia & 118,63 & 84,20 & 103,01 & 106,98 \\
\hline Anóxica & 68,45 & 52,38 & 56,11 & 61,33 \\
\hline Sedimentación & 60,81 & 50,83 & 53,81 & 53,16 \\
\hline
\end{tabular}

carbono orgánico con el que se garantiza el proceso de desnitrificación anóxica [24]. En la Figura 8, se observa el comportamiento de los parámetros fisicoquímicos analizados, para el ciclo D en fase de operación normal.

En la Figura 8a se observó un descenso de la concentración de materia orgánica en las dos

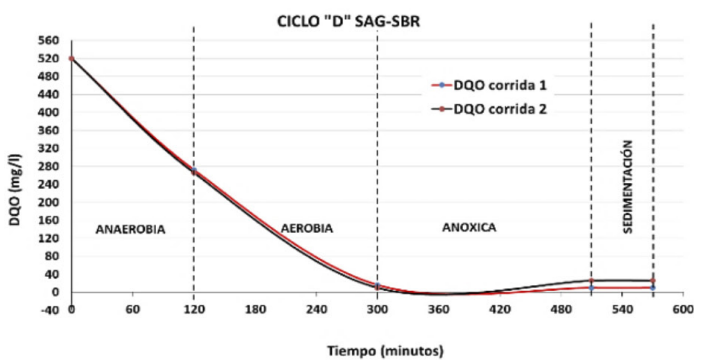

(a)

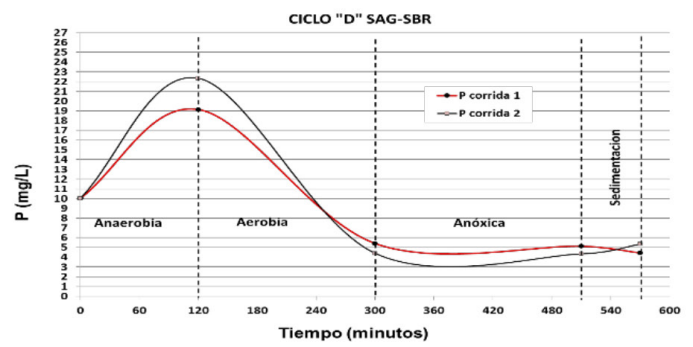

(b)

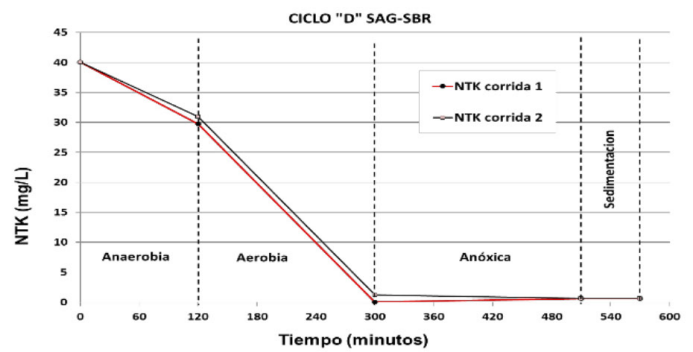

(c)

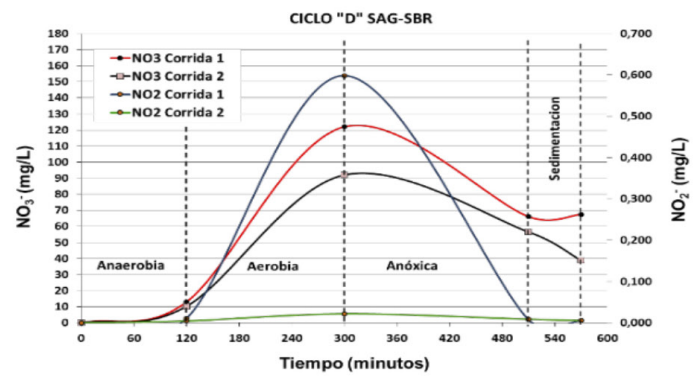

(d)

Figura 8. Variación de: (a) DQO, (b) P, (c) NTK, (d) $\mathrm{NO}_{3}{ }^{-}$y $\mathrm{NO}_{2}{ }^{-}$en SAG-SBR en operación normal para iclo $\mathrm{D}$.

primeras fases: anaeróbica y aeróbica, en las que existe un consumo importante de carbono inorgánico y orgánico, pues como se precisará más adelante, en estos períodos de aproximadamente 300 minutos aconteció la nitrificación-desnitrificación simultánea (NDS). 
En la Figura 8b, se observó la liberación y extraasimilación de $P$ en fases anaeróbica y aeróbica, con un comportamiento que fue creciente en términos de eliminación de P hacia los TRH de menor duración, en particular para el ciclo D que correspondió a 9,5 h en total, en el que se alcanzó una eliminación total de $51,27 \%$. Lo anterior indica que la eliminación del P mejoró su desempeño, considerando la complementariedad en términos de diversidad de poblaciones microbianas que mantienen la biomasa suspendida y adherida (biopelícula) generada en los medios de contacto móvil y fijo, que fueron integrados para el tratamiento en el reactor SAGSBR. Se infiere que esta condición fue favorecida considerando que durante el metabolismo anaeróbico, a bajos tiempos de retención hidráulica, se pudo disminuir la competencia por sustrato fácilmente biodegradable presente en el ARS empleada en la investigación, entre los organismos acumuladores de fósforo (PAO) y los acumuladores de glucógeno (GAO), con concentraciones limitadas de ácidos grasos volátiles (AGV) de cadena corta aportados por el ácido acético (véase Tabla 2); estos últimos microorganismos, no liberan ni extraasimilan $\mathrm{P}$, sin brindar aportes importantes en el proceso [25-27]. Esta actividad en la eliminación de P se logra por la actividad de los organismos PAO, alternando condiciones anaerobias/anóxicas y aerobias [28].

También se registró la eficiencia del proceso de nitrificación, donde el NTK se redujo a niveles muy bajos en la fase aerobia, alcanzando valores cercanos a cero $(0,0)$; lo que representa una eliminación de NTK por encima de $96 \%$, logrando un máximo de 96,6\% en el ciclo D del SAG-SBR; esta condición demuestra el desarrollo óptimo de las bacterias nitrificantes, responsables de oxidar el nitrógeno orgánico y el $\mathrm{NH}_{4}{ }^{+} \mathrm{a} \mathrm{NO}_{2}^{-}$y $\mathrm{NO}_{3}^{-}$, condición que se fundamenta en el hecho de combinar el crecimiento biológico suspendido con el adherido que promueve el desarrollo de biopelícula, la que genera mejores tasas de nitrificación en la superficie de los medios de contacto, con un ambiente y hábitat propicio para su metabolismo y desarrollo [11, 29-31].

El análisis de resultados y del balance de masas efectuado en términos del nitrógeno total (NT) para las corridas de los ciclos efectuados en el SAG-SBR mostraron que en la salida de la fase aerobia se presentó la desnitrificación aerobia, en donde buena parte del contenido de $\mathrm{N}$ fue liberado en forma de gas. Se infiere que este proceso de eliminación de nitrógeno ocurrió por la formación de microzonas anóxicas en la biopelícula formada en los medios de contactos fijos y móviles [32]. La nitrificación ocurrió en la superficie de la biopelícula mientras que la desnitrificación se desarrolló en las capas internas, debido al gradiente de OD dentro de la biopelícula [33]. En términos generales, los valores de $\mathrm{NO}_{3}^{-}$, en el ciclo $\mathrm{D}$, alcanzaron valores entre $92 \mathrm{mg} / \mathrm{l} \mathrm{y}$ 122 mg/l (véase Figura 8d), concentraciones que pudieron ser más altas, sino se hubiese llevado a cabo la NDS aerobia, condición que fue probada al realizar el respectivo balance de NT en el proceso.

Tabla 7. Valores promedio de sólidos en el Reactor SAG-SBR en operación normal.

\begin{tabular}{|l|c|c|c|c|c|c|}
\hline \multirow{2}{*}{ Fase } & \multicolumn{6}{|c|}{ Promedios en sólidos (mg/l) } \\
\cline { 2 - 7 } & \multicolumn{5}{|c|}{ A } & \multicolumn{3}{c|}{ B } \\
\cline { 2 - 7 } & ST & SST & SSV & ST & SST & SSV \\
\hline Anaerobia & 3.400 & 2.175 & 2.001 & 3.390 & 2.120 & 2.003 \\
\hline Aerobia & 3.605 & 2.770 & 2.460 & 3.860 & 2.780 & 2.562 \\
\hline Anóxica & 3.000 & 2.120 & 2.003 & 2.800 & 2.090 & 1.985 \\
\hline Sedimentación & 206 & 23 & 19 & 320 & 38 & 30 \\
\hline Eliminación \% & 94,29 & 99,17 & 99,22 & 91,71 & 98,63 & 98,81 \\
\hline & \multicolumn{7}{|c|}{ C } & & \multicolumn{3}{|c|}{ D } & \\
\hline Anaerobia & 2860 & 2.190 & 2.067 & 2.965 & 2.507 & 2.135 \\
\hline Aerobia & 3500 & 3.100 & 2.634 & 3.600 & 3.210 & 2.646 \\
\hline Anóxica & 3100 & 2.620 & 2.199 & 3.060 & 2.550 & 2.213 \\
\hline Sedimentación & 180 & 32 & 26 & 165 & 31 & 26 \\
\hline Eliminación \% & 94,86 & 98,97 & 99,00 & 95,42 & 99,03 & 99,00 \\
\hline
\end{tabular}


En lo correspondiente a la eliminación de sólidos (ST, SST y SSV), en la Tabla 7 y Figura 9, se presentan los resultados obtenidos de acuerdo con los ciclos empleados. Los valores en sólidos fueron obtenidos en el licor mezcla para la etapa anaerobia, óxica y anóxica y en el clarificado para la sedimentación. En la sedimentación se lograron importantes remociones en todos los tipos de sólidos y su cálculo se efectuó con respecto a la fase aeróbica, por ser en esta donde se obtiene mayor producción de lodos.

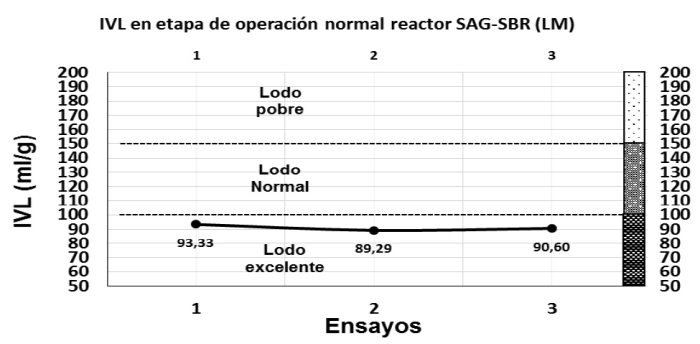

(a)

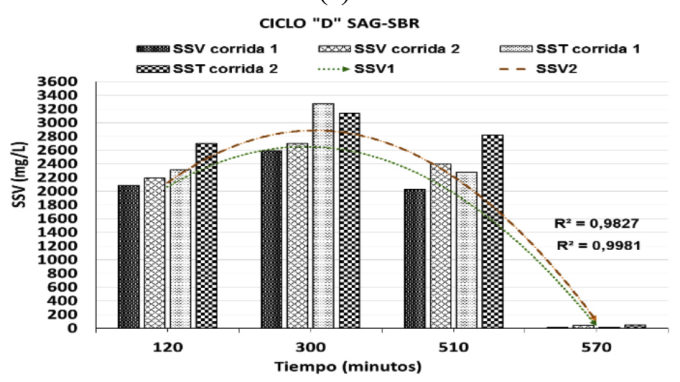

(b)

Figura 9. (a) Comportamiento de: (a) IVL; (b) sólidos en ciclo D SAG-SBR en operación normal.

Asimismo, la biomasa se incrementó en la salida de la fase aerobia debido al metabolismo respiratorio para la síntesis celular. La eliminación de estos fue evidente, considerando las buenas características de sedimentabilidad (IVL) del lodo con el que se operó el sistema. Esta situación se puede corroborar por las bajas concentraciones en el clarificado, en particular de SSV donde las concentraciones finales fueron inferiores a $30 \mathrm{mg} / \mathrm{l}$ y para los sólidos suspendidos menores a $40 \mathrm{mg} / \mathrm{l}$.

Con base en los resultados indicados en la Tabla 8 y en la Figura 10, se logró establecer la complementariedad que ofrece el tratamiento en biopelícula al medio biológico suspendido en una secuencia AOA, en particular con la eliminación del NT. Igualmente, es importante resaltar el beneficio asociado al buen rendimiento en la eliminación del NT, generado por desnitrificación directa en fase aerobia, mediante el proceso NDS.

La tendencia es clara al incrementarse los niveles de remoción de MO, P y NT, cuando bajan los tiempos de retención hidráulico de la secuencia AOA, en particular para la distribución establecida en el ciclo $\mathrm{D}$, con el que se lograron porcentajes de remoción de $96,62 \%, 51,27 \%$ y $68,62 \%$, para MO, P y N, respectivamente.

Tabla 8. Promedios de eliminación simultánea de MO, NT y P del SAG-SBR en operación normal.

\begin{tabular}{|c|c|c|c|}
\hline \multicolumn{4}{|c|}{ Reactor SAG-SBR (\% de eliminación) } \\
\hline Parámetro & MO & NT & P \\
\hline Ciclo A & 96,31 & 59,55 & 34,20 \\
\hline Ciclo B & 98,15 & 66,20 & 33,38 \\
\hline Ciclo C & 96,92 & 68,20 & 40,14 \\
\hline Ciclo D & 96,62 & 68,62 & 51,27 \\
\hline
\end{tabular}

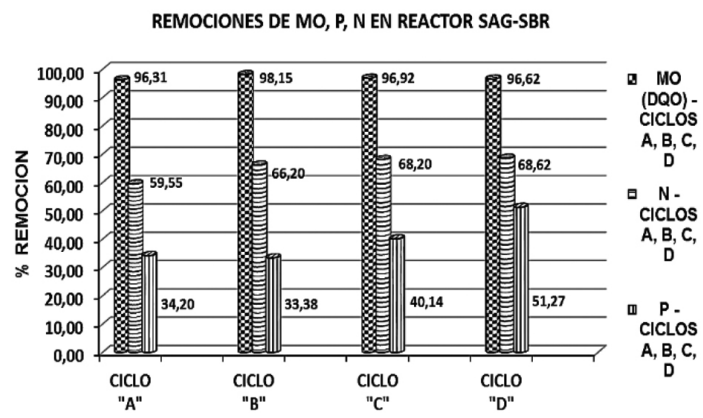

Figura 10. Remoción de MO, P y NT del SAG-SBR en operación normal.

El diseño propio del reactor SBR empleado en esta investigación, el cual integró el tratamiento de la ARS con medios de contacto móviles y fijos en una secuencia AOA, y la NDS en fase aerobia que ha ganado importancia en los últimos años en la remoción de $\mathrm{N}$ [34]; permitió el proceso de tratamiento gracias al hábitat propicio que generó el tratamiento biológico bajo la combinación simultánea de lechos de biomasa suspendida y adherida [17]. 
Esta característica fue prácticamente similar para todos los ciclos evaluados, aunque, fue más eficaz cuando los períodos de duración de la fase aerobia en los ciclos fueron del orden de 4 horas, disminuyendo un poco hacia 3,5 y 3 horas de duración.

No obstante lo anterior, en términos de remoción simultánea de MO, P y NT fue el ciclo D, el que representó las mayores tasas de remoción de estos contaminantes, representando una remoción de NT de $68,62 \%$, de los cuales por NDS se eliminó el 37,92\% en fase aerobia y el restante por desnitrificación anóxica. Este fenómeno se puede evidenciar en la Figura 11, donde se observa el promedio obtenido en fase aerobia para los ciclos evaluados.

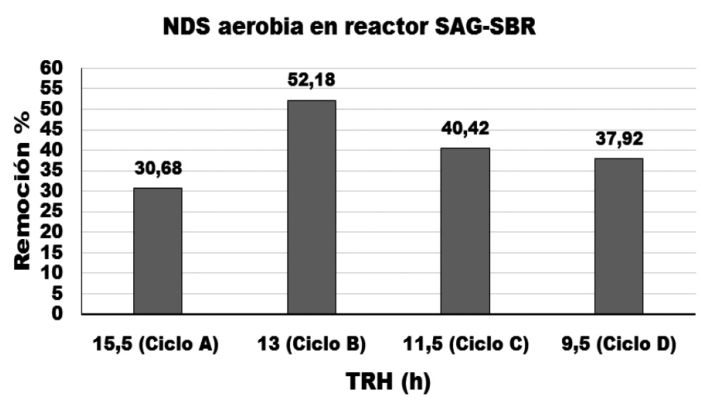

Figura 11.NDS en fase aerobia del SAG-SBR operación normal.

\section{CONCLUSIONES}

La estabilización del reactor SAG-SBR a escala laboratorio, se logró en un término de 2,5 meses obteniendo lodos biológicos con índices volumétricos entre 91 y $93 \mathrm{ml} / \mathrm{g}$ y alcanzado a su vez eliminaciones en MO, NT y P de $97,53 \%, 63,25 \%$ y $34,13 \%$, respectivamente.

Con la presente investigación se logró corroborar que la tecnología SBR presenta versatilidad frente a las diferentes modificaciones y adaptaciones en su régimen de operación, encontrándose a escala de laboratorio que la combinación de fases en una secuencia anaeróbica-óxica-anóxica (AOA), soportada en el metabolismo simultáneo de biomasa activa suspendida y adherida en medios de contacto móviles y fijos, incrementó la eficiencia en la eliminación simultánea de MO, NT y P, particularmente hacia los ciclos con menores TRH, como fue el ciclo "D" de 9,5 horas con el que se logró una eliminación simultánea de 96,62\%, 68,62\% y $51,27 \%$, respectivamente.

Se logró la eliminación eficaz de sólidos asociada a la óptima conformación y mantenimiento del flóculo en el licor mezcla, quien registró valores de IVL promedio de $91,07 \mathrm{ml} / \mathrm{g}$, y para el ciclo D, se alcanzaron eliminaciones del 95,42\%, 99,03\% y 99\% para ST, SST y SSV, respectivamente.

Con base en el valor promedio obtenido en los ciclos evaluados para la fase aerobia, la nitrificación desnitrificación simultánea (NDS) se presentó eficazmente en la secuencia AOA adoptada para el régimen de trabajo del SAG-SBR, logrando complementar la desnitrificación anóxica en la remoción de NT.

\section{AGRADECIMIENTOS}

Esta investigación se realizó con el apoyo técnico y administrativo de la Corporación Autónoma Regional de Nariño CORPONARIÑO y de la Universidad Mariana (Facultad de Posgrados y Relaciones Internacionales, Facultad de Ingeniería y Laboratorio de Ingeniería Ambiental); igualmente gracias a la asesoría brindada por la Universidad de Antioquia.

\section{REFERENCIAS}

[1] M. González y J. Saldarriaga. "Remoción biológica de materia orgánica, nitrógeno y fósforo en un sistema tipo anaerobio-anóxicoaerobio". Revista EIA. No 10, pp. 46-53. Enero 2008. ISSN: 1794-1237. URL: http:// www.scielo.org.co/scielo.php?script=sci_ar ttext\&pid=S1794-12372008000200005

[2] L. Rosell. "Evaluación de la Calidad de las Aguas de La Bahía de Bluefields (Raas, Nicaragua)". Tesis para optar al grado de Magíster. Universidad Politécnica de Catalunya. Barcelona, España. 2009.

[3] J.F. Muñoz y R.M. Ramos. "Reactores Discontinuos Secuenciales: una Tecnología Versátil en el Tratamiento de Aguas Residuales". Ciencia e Ingeniería Neogranadina. Vol. 24, $\mathrm{N}^{\circ}$ 1, pp. 49-66. Junio 2014. ISSN: 01248170. URL: http://www.scielo.org.co/pdf/ cein/v24n1/v24n1a03.pdf 
[4] E. Sperling, A.C. Ferreira and L. Gomes. "Comparative eutrophication development in two Brazilian water supply reservoirs with respect to nutrient concentrations and bacteria growth". Desalination. Vol. 226, Issues 1-3, pp. 169-174. June 2008. ISSN: 0011-9164.URL: http://www.sciencedirect. com/science/article/pii/S0011916408001483. DOI: 10.1016/j.desal.2007.02.105

[5] Y. Rahimi, A. Torabian, N. Mehrdadi and B. Shahmoradi. "Simultaneous Nitrificationdenitrification and Phosphorus Removal in a Fixed Bed Sequencing Batch Reactor (FBSBR)". Journal of Hazardous Materials Vol. 185, Issues 2-3, pp. 852-857. January, 2011. ISSN: 1873-3336. URL: http:// www.sciencedirect.com/science/article/ pii/S0304389410012598. DOI: 10.1016/j. jhazmat.2010.09.098

[6] Y. Avnimelech. "Bio-filters: The Need for a New Comprehensive Approach". Vol. 34, Issue 3, pp. 172-178. May 2006. ISSN: 01448609. URL: http://www.sciencedirect.com/ science/article/pii/S0144860905000361. DOI: 10.1016/j.aquaeng.2005.04.001

[7] L. Fewtrell. "Drinking-Water Nitrate, Methemoglobinemia, and Global Burden of Disease: A Discussion". Environmental Health Perspectives, Vol. 112 N$^{\circ}$ 14, pp. 13711374. July, 2004. ISSN: 0091-6765. URL: http://www.ncbi.nlm.nih.gov/pmc/articles/ PMC1247562/ DOI: 10.1289/ehp.7216

[8] J.A. Camargo and A. Alonso. "Ecological and Toxicological Effects of Inorganic Nitrogen Pollution in Aquatic Ecosystems: a Global Assessment". Environment International. Vol. 32, Issue 6, pp. 831-849. August, 2006. ISSN: 0160-4120. URL: http:// www.sciencedirect.com/science/article/ pii/S0160412006000602. DOI: 10.1016/j. envint.2006.05.002

[9] M. Casais y G. Rosa. "Riesgos de la preparación colónica con fosfato sódico". Revista Argentina de Anestesiología. Vol. 68, $\mathrm{N}^{\circ}$ 2, pp. 203-211. Mayo 2010. ISSN: 03707792. URL: http://www.anestesia.org.ar/ search/articulos_completos/1/1/1328/c.pdf

[10] Environmental Protection Agency (EPA). "Water Quality Standards for Coastal and Great Lakes Recreation Waters". November, 2004. Date of visit: December, 2014. URL: http://water.epa.gov/lawsregs/lawsguidance/ beachrules/upload/2004_11_16_2004_ November_Day-16_w25303.pdf

[11] J. Escobar. "La contaminación de los ríos y sus efectos en las áreas costeras y el mar". Naciones Unidas - CEPAL, Santiago de Chile, Chile. 2002. Fecha de consulta: Diciembre 2014. URL: http://www.bvsde.paho.org/ bvsacd/aidis-ar/lcl1799e.pdf

[12] C. Venegas, J. Manga, D. Abad y J. Ribes. "Efecto del número de ciclos anóxicos / aerobios sobre la eliminación biológica de nitrógeno en un sistema de fangos activados en discontinuo (SBR)". Ingeniería y Desarrollo. No 15, pp. 45-56. 2004. ISSN: 2145-9371.

[13] J. Palma y J. Manga. "Simulación de un sistema de fangos activados en discontinuo (SBR) para el tratamiento de aguas residuales con altos contenidos de nitrógeno". Ingeniería y Desarrollo. No 18, pp. 61-71. 2005. ISSN: 2145-9371.

[14] M.T. Vives. "SBR Technology for Wastewater Treatment: Suitable Operational Conditions for a Nutrient Removal". Tesis para optar al grado de Doctor. Universidad de Girona. Girona, España. 2004.

[15] Y. Jin, D. Ding, C. Feng, S. Tong, T. Suemura and F. Zhang. "Performance of sequencing batch biofilm reactors with different control systems in treating synthetic municipal wastewater". Bioresource Technology. Vol. 104, pp. 12-18. January, 2012. ISSN: 09608524. URL: http://www.sciencedirect.com/ science/article/pii/S0960852411011965?np=y. DOI: 10.1016/j.biortech.2011.08.086

[16] C. Wu and Y. Jin. "Performance of Nitrogen and Phosphorus Removal of Municipal Wastewater in Sequencing Batch Biofilm Reactor". Energy Procedia. Vol. 11, pp. 44534457. March 2011. ISSN: 1876-6102. URL: https://www.infona.pl/resource/bwmeta1. element.elsevier-ee11bdfb-a28b-34b789a5-e50103094893. DOI: 10.1016/j. egypro.2011.10.860.

[17] A. Gieseke, P.R. Amann and A. Schramm. "Simultaneous $\mathrm{P}$ and $\mathrm{N}$ removal in a sequencing batch biofilm reactor: insights from reactor and microscale investigations". Water Research. Vol. 36, Issue 2, pp. 501509. January, 2002. ISSN: 0043-1354. URL: http://www.sciencedirect.com/science/article/ 
pii/S0043135401002329. DOI: 10.1016/ S0043-1354(01)00232-9.

[18] APHA-AWWA-WEF. "Standard Methods for the Examination of Water and Wastewater". 21st edition, pp. 1368. Washington, USA. 2005. ISBN: 0875530478.

[19] J.C. Saldarriaga, J.M. Garrido y D.A. Hoyos. "Remoción Simultánea de Carbono, Nitrógeno y Fósforo de Aguas Residuales en un Sistema Híbrido UCT Modificado". Dyna. Vol. 162, pp. 39-48. Junio 2010. ISSN: 2346-2183. URL: http://www.revistas.unal. edu.co/index.php/dyna/article/view/15674.

[20] G. Smolders, J. Van der Meij, M. Van Loosdrecht and J. Heijnen. "Stoichiometric model of the aerobic metabolism of the biological phosphorus removal process". Biotechnology and Bioengineering. Vol. 44, Issue 7, pp. 837-848. September, 1994. ISSN: 0006-3592. URL: http://onlinelibrary.wiley. com/doi/10.1002/bit.260440709/abstractDOI: 10.1002/bit.260440709

[21] Metcalf and Eddy. "Wastewater Engineering: Treatment and Reuse". McGraw-Hill. $5^{\text {th }}$ edition, pp. 1856. New York, USA. 2013. ISBN: 978-1259010798.

[22] M. Gerardi. "Nitrification and denitrification in the activated sludge process", pp. 208. John Wiley \& Sons. New York, USA. 2002. ISBN: 978-0471065080.

[23] Y. Jin and C. Wu. "Influence of $\mathrm{C} / \mathrm{N}$ Ratio on Nitrogen Removal in Sequencing Batch Biofilm Reactor". 5th International Conference on Bioinformatics and Biomedical Engineering, pp. 1-4. May, 2011. ISSN: 21517614. URL: http://ieeexplore.ieee.org/xpl/ articleDetails.jsp?arnumber $=5781048$ DOI: 10.1109/icbbe. 2011.5781048

[24] A.H. Mahvi. "Sequencing batch reactor: a promising technology in wastewater treatment". Iranian Journal of Environmental Health, Science and Engineering. Vol. 5 $\mathrm{N}^{\circ} 2$, pp. 79-90. March, 2008. ISSN: 2052336X. URL: http://ijehse.tums.ac.ir/index. php/ijehse/article/view/153/152

[25] L.-M. Whang and J.K. Park. "Competition between polyphosphate and glycogen accumulating organisms in enhancedbiological phosphorus removal systems: Effect of temperature and sludge age". Water Environmental Research.
Vol. $78 \mathrm{~N}^{\mathrm{o}}$ 1, pp. 4-11. January, 2006. ISSN: 1554-7531. DOI: http://dx.doi. org/10.2175/106143005X84459. URL: http://www.ingentaconnect.com/contentone/ wef/wer/2006/00000078/00000001/art00002

[26] L.M. Whang, C.D.M. Filipe and J.K. Park. "Model-based evaluation of competition between polyphosphate- and glycogenaccumulating organisms". Water research. Vol. 41, Issue 6, pp. 1312-1324. March 2007. ISSN: 0043-1354. URL: http:// www.sciencedirect.com/science/article/pii/ S0043135406007056. DOI: doi:10.1016/j. watres.2006.12.022

[27] A. Oehmen, A.M. Saunders, M.T. Vives, Z. Yuan and J. Keller. "Competition between polyphosphate and glycogen accumulating organisms in enhanced biological phosphorus removal systems with acetate and propionate as carbon sources". Journal of Biotechnology. Vol. 123, Issue 1, pp. 22-32. May, 2006. ISSN: 0168-1656. URL: http://www.sciencedirect.com/science/ article/pii/S0168165605006565. DOI: 10.1016/j.jbiotec.2005.10.009

[28] S. Abualhail, R. Naseer and L. Xiwu. "Biological nutrient removal using a novel five-step continuous flow activated sludge process technology". Environment Protection Engineering. Vol. $39 \mathrm{~N}^{\mathrm{o}}$ 3, pp. 165-184. ISSN: 2450-260X. URL: http://epe.pwr. wroc.pl/2013/3-2013/Abualhail_3-2013. pdf DOI: $10.5277 /$ epe 130312

[29] Y. Rahimi, A. Torabian, N. Mehrdadi and B. Shahmoradi. "Simultaneous nitrificationdenitrification and phosphorus removal in a fixed bed sequencing batch reactor (FBSBR)". Journal of Hazardous Materials. Vol. 185, Issues 2-3, pp. 852-857. January, 2011. ISSN: 0304-3894. DOI: 10.1016/j. jhazmat.2010.09.098. URL: http://www. sciencedirect.com/science/article/pii/ S0304389410012598

[30] G. Ferrara and F. Rodríguez. "Biological nitrogen removal using combinated fixedfilm and suspended reactors by intermittent operation". Revista Técnica Facultad de Ingeniería. Vol. $32 \mathrm{~N}^{\circ} 3$, pp. 222-230. Diciembre 2009. ISSN: 0254-0770. URL: http://www.produccioncientificaluz.org/ index.php/tecnica/article/view/6679 
[31] D.S. Kim, N.S. Jung and Y.S Park. "Characteristics of nitrogen and phosphorus removal in SBR and SBBR with different ammonium loading rates". Korean Journal of Chemical Engineering. Vol. 25, Issue 4, pp. 793-800. July, 2008. ISSN: 1975-7220. URL: http://link. springer.com/article/10.1007/s11814-0080130-2. DOI: 10.1007/s11814-008-0130-2

[32] P. Wilderer, P. Arnz and E. Arnold. "Application of biofilms and biofilm support materials as a temporary sink and source". Water air and Soil Polution, Vol. 123, Issue 1-4, pp. 147-158. October, 2000. ISSN: 15732932. URL: http://link.springer.com/article /10.1023\%2FA\%3A1005237617334. DOI: 10.1023/A:1005237617334.
[33] D.M. White, T.A Pilon and C. Woolard. "Biological treatment of cyanide containing wastewater". Water Research. Vol. 34, Issue 7, pp. 2105-2109. May, 2000. ISSN: 0043-1354. URL: http://www.sciencedirect. com/science/article/pii/S0043135499003620. DOI: 10.1016/S0043-1354(99)00362-0

[34] R.R. Eldon, J.K. Sung and S.P. Hung. "Effect of COD/N ratio and salinity on the performance of sequencing batch reactors". Bioresource Technology. Vol. 99, Issue 4, pp. 839-846. March, 2008. ISSN: 0960-8524. DOI: 10.1016/j.biortech.2007.01.037. URL: http://www.sciencedirect.com/science/article/ pii/S0960852407000971 